\title{
Theory of the Lattice Boltzmann Equation: Symmetry properties of discrete velocity sets
}

\author{
Robert Rubinstein ${ }^{1, *}$ and Li-Shi $\mathrm{Luo}^{2, \dagger}$ \\ ${ }^{1}$ NASA Langley Research Center, Hampton, Virginia 23681, USA \\ ${ }^{2}$ Department of Mathematics and Statistics, Old \\ Dominion University, Norfolk, Virginia 23529, USA
}

(Dated: February 1, 2007)

\begin{abstract}
In the lattice Boltzmann equation, continuous particle velocity space is replaced by a finite dimensional discrete set. The number of linearly independent velocity moments in a lattice Boltzmann model cannot exceed the number of discrete velocities. Thus, finite dimensionality introduces linear dependencies among the moments that do not exist in the exact continuous theory. Given a discrete velocity set, it is important to know to exactly what order moments are free of these dependencies. Elementary group theory is applied to the solution of this problem. It is found that by decomposing the velocity set into subsets that transform among themselves under an appropriate symmetry group, it becomes relatively straightforward to assess the behavior of moments in the theory. The construction of some standard two- and three-dimensional models is reviewed from this viewpoint, and procedures for constructing some new higher dimensional models are suggested.
\end{abstract}

PACS numbers: 47.10.-g, 47.11.-j, 02.20.-a

*Electronic address: r.rubinstein@larc.nasa.gov

${ }^{\dagger}$ Electronic address: lluo@odu.edu 


\section{INTRODUCTION}

A guiding principle of this series of papers is the formulation of the lattice Boltzmann equation (LBE) as a discrete kinetic theory [1-10]. In kinetic theory, moments of the distribution function $f(\boldsymbol{x}, \boldsymbol{\xi}, t)$ over the space of velocities $\boldsymbol{\xi}$ are tensors of various ranks $[11,12]$ that define continuum fluid properties including mass, momentum, energy, stress, and heat flux. Analogous moments can be formed over a finite velocity space in the discrete setting of the lattice Boltzmann equation; however, discreteness permits degeneracies that do not exist in the continuous case [13].

Thus, in kinetic theory, the number of linearly independent components of the general moment of order $n$,

$$
\mathrm{M}(\boldsymbol{x}, t)=\int d \boldsymbol{\xi} \underbrace{\boldsymbol{\xi} \cdots \boldsymbol{\xi}}_{n \text { times }} f(\boldsymbol{x}, \boldsymbol{\xi}, t)
$$

is equal to the number of linearly independent products $\boldsymbol{\xi} \cdots \boldsymbol{\xi}$ in the integrand, namely $(n+1)(n+2) / 2$ in three-dimensional configuration space, and $(n+1)$ in two dimensions. But in a discrete kinetic theory, in which the integral over continuous velocity space $\boldsymbol{\xi}$ in Eq. (1) is replaced by a sum over a finite velocity set $\mathrm{C}$ with $N$ elements,

$$
\mathrm{M}(\boldsymbol{x}, t)=\sum_{\boldsymbol{c}_{i} \in \mathrm{C}} \underbrace{\boldsymbol{c}_{i} \cdots \boldsymbol{c}_{i}}_{n \text { times }} f\left(\boldsymbol{x}, \boldsymbol{c}_{i}, t\right)=\sum_{\boldsymbol{c}_{i} \in \mathrm{C}} \underbrace{\boldsymbol{c}_{i} \cdots \boldsymbol{c}_{i}}_{n \text { times }} f_{i}(\boldsymbol{x}, t)
$$

the factor $f_{i}(\boldsymbol{x}, t):=f\left(\boldsymbol{x}, \boldsymbol{c}_{i}, t\right)$ restricts the number of linearly independent components to at most $N$. Therefore, if $N<(n+1)(n+2) / 2$, the moment in the discrete theory defined by Eq. (2) necessarily has fewer independent components than its continuous counterpart in Eq. (1). But such a reduction remains possible due to linear dependencies even if $N \geq$ $(n+1)(n+2) / 2$. Whenever a moment in a discrete theory has fewer independent components than its continuous counterpart, we will say that the moment is incomplete in this discrete theory; if it has the same number, we will say that it is complete.

Since incomplete moments in a discrete model are artifacts without continuous analogs, a fundamental problem in discrete kinetic theory is to determine what moments are complete for a given finite velocity set $C:=\left\{\boldsymbol{c}_{i}\right\}$ : a realistic description by a discrete theory is possible only for complete moments. The purpose of this paper is to develop a systematic approach to solving this problem. It is based on the observation that the discrete velocity set $\left\{\boldsymbol{c}_{i}\right\}$ is not an arbitrary collection of vectors, but is chosen to be as symmetric as possible so that the 
model can mimic the physical isotropy of the fluid. In particular, two-dimensional models are typically constructed so that the discrete velocity set has the symmetry of a square: that is, it is invariant under symmetries of two-dimensional $\boldsymbol{\xi}$-space that map the set of four velocities $\{( \pm 1, \pm 1)\}$ into itself, and three-dimensional models are typically constructed so that the velocity set has the symmetry of a cube: that is, it is invariant under symmetries of three-dimensional $\boldsymbol{\xi}$-space that map the analogous set of eight velocities $\{( \pm 1, \pm 1, \pm 1)\}$ into itself.

It is natural to exploit the symmetry of the configuration of discrete velocities by applying the elementary representation theory of finite groups (cf. $[14,15])$. Although models can and have been constructed case-by-case using elementary methods, group theory offers the advantages of a systematic and unified approach. We do not claim that it results in a 'better' way to construct models, in the sense of being faster, easier to formulate, or even easier to understand. We only contend that it is a natural approach that reveals some problems common to constructing all discrete models, which can be hidden by lengthy algebra, even (perhaps especially) if the algebra is done symbolically. We note that group theory has also been used to study the lattice-gas cellular automata [16-19] and the lattice Boltzmann models [20-23] from a different perspective.

The content of this paper is limited to the kinematics of the description of moments by finite discrete-velocity models; a moment need not be well described dynamically in a model in which it is complete: This issue will be discussed briefly at the end of the paper. An outline of the paper is as follows. We shall first review elementary group theory and its application to simple discrete velocity sets in three dimensions based on the vertices, edges, and faces of a cube. The group theoretical analysis will be used to study models D3Q6, D3Q13, D3Q15, D3Q19, and D3Q27. (Here, the standard notation DdQq is used to denote a $q$-velocity model in $d$ dimensional space.) Some preliminary discussion of a D3Q51 model will be given. We shall also briefly discuss some two-dimensional models. It will be shown that expressing the models in terms of irreducible representations (cf. [14, 15, 24]) of the the group of symmetries of a cube or a square can help answer many basic questions about the kinematics of discrete models. Brief discussions are then given of the role of the choice of symmetry group and of the role of higher order moments in discrete hydrodynamics. 


\section{DECOMPOSITION INTO IRREDUCIBLE REPRESENTATIONS IN 3D}

The formulation of lattice Boltzmann models begins with a discrete velocity set $C=\left\{\boldsymbol{c}_{i}\right\}$ chosen from a lattice $\delta_{x} \mathbb{Z}^{D}$ with lattice constant $\delta_{x}$ in continuous $D$-dimensional velocity space $\boldsymbol{\xi}$. In three dimensions, the highest symmetry possible for the set $\mathrm{C}$ is the symmetry of a cube (cf. [24]). Since a model with any less symmetry cannot be satisfactory, this symmetry will be imposed on all velocity sets $C$ at the outset. We will describe this symmetry by the group of 24 rotations of the cube. Adding inversions leads to the complete group of 48 symmetries; however, it will appear that using this larger group does not lead to anything essentially new, and that the main ideas can be explained and understood more simply using the smaller group.

The distribution function is a finite sum

$$
f(\boldsymbol{x}, \boldsymbol{\xi}, t):=\sum_{\boldsymbol{c}_{i} \in \mathrm{C}} f_{i}(\boldsymbol{x}, t) \delta\left(\boldsymbol{\xi}-\boldsymbol{c}_{i}\right),
$$

where $f_{i}(\boldsymbol{x}, t):=f\left(\boldsymbol{x}, \boldsymbol{c}_{i}, t\right)$ defines the number density of particles with velocity $\boldsymbol{c}_{i}$. The multiple relaxation time formalism of d'Humières $[5,13,25]$ models the collision process through the relaxation of moments, which therefore play a central role in the formulation. Moments of the distribution function $f$ are defined in terms of polynomials chosen from a set $\left\{p_{j}(\boldsymbol{\xi})\right\}$ by

$$
\mathrm{M}_{j}(\boldsymbol{x}, t)=\int d \boldsymbol{\xi} f(\boldsymbol{x}, \boldsymbol{\xi}, t) p_{j}(\boldsymbol{\xi})=\sum_{\boldsymbol{c}_{i} \in \mathrm{C}} f_{i}(\boldsymbol{x}, t) p_{j}\left(\boldsymbol{c}_{i}\right) .
$$

Thus, values of moments are determined by linear combinations of rows of the matrix

$$
A_{i j}=\left\langle p_{j} \mid \boldsymbol{c}_{i}\right\rangle:=p_{j}\left(\boldsymbol{c}_{i}\right)
$$

where the bra-ket notation denotes evaluation of the left-hand member (a polynomial $p_{j}$ ) on the right-hand member (a point $\boldsymbol{c}_{i}$ ) extended by linearity to linear combinations of velocities and polynomials. Note that the moment defined in Eq. (4) can be understood as a weighted sum of values of the distribution function $f_{i}$, with weights $p_{j}\left(\boldsymbol{c}_{i}\right)$.

The number of linearly independent moments of order $n$ is simply the rank of the matrix A in Eq. (5) when $p_{j}$ varies over the homogeneous polynomials of degree $n$. The rank can certainly be found by straightforward linear algebra; however, useful simplifications result if $\mathrm{A}$ is evaluated after expressing both the velocity set $\left\{\boldsymbol{c}_{i}\right\}$ and the polynomial set $\left\{p_{j}(\boldsymbol{\xi})\right\}$, regarded as bases of vector spaces on which the group of rotations of the cube acts as a group 
of linear transformations, in bases $\left\{\tilde{\boldsymbol{c}}_{i}\right\}$ and $\left\{\tilde{p}_{j}(\boldsymbol{\xi})\right\}$ consisting of quantities that transform by irreducible representations $[14,15,24]$ of the group of rotations of the cube. The resulting matrix will be denoted by $\tilde{A}$. The irreducible representations are the representations of lowest dimension from which all other representations can be constructed; we refer to elementary texts (e.g., $[14,15,24])$ for details. Fundamental orthogonality properties $[14,15,24]$ imply that $\tilde{A}_{i j}=\left\langle\tilde{p}_{j} \mid \tilde{\boldsymbol{c}}_{i}\right\rangle=0$ whenever $\tilde{p}_{j}$ and $\tilde{\boldsymbol{c}}_{i}$ belong to different irreducible representations. Thus, $\tilde{\mathrm{A}}$ consists of blocks $\tilde{A}_{i j}$ in which indices $i$ and $j$ vary over vectors transforming by the same irreducible representation. These blocks are $d n \times d n$ if there are $n$ occurrences of an irreducible representation of dimension $d$; since these blocks are much smaller than the original matrix $A$, the computation of the rank is much simpler.

The irreducible representations of the group of 24 rotations of the cube are exhibited in the following table $[14,15,24]$ :

\begin{tabular}{l|l}
\hline repr. & \multicolumn{1}{|c}{ polynomials } \\
\hline$\Gamma_{1}$ & 1 \\
$\Gamma_{1}^{\prime}$ & $x y z$ \\
$\Gamma_{2}$ & $\left\{\left(x^{2}-y^{2}\right),\left(y^{2}-z^{2}\right),\left(z^{2}-x^{2}\right)\right\}:=\left\{*\left(x^{2}-y^{2}\right)\right\}$ \\
$\Gamma_{3}$ & $\{x y, y z, z x\}:=\{* x y\}$ \\
$\Gamma_{3}^{\prime}$ & $\{x, y, z\}:=\{* x\}$ \\
\hline
\end{tabular}

The $*$ notation will be used henceforth to indicate the additional polynomials obtained by cyclic permutation of $x, y$, and $z$ : we use $x, y$, and $z$, or index notation $x_{i}, i \in\{x, y, z\}:=$ $\{1,2,3\}$, as convenient, to denote the components of the particle velocity $\boldsymbol{\xi}$ : no ambiguity is possible because configuration space $\boldsymbol{x}$ plays no role in this paper. For each representation $\Gamma_{n}$, the subscript $n$ indicates its dimension, and a set of $n$ linearly independent polynomials is given which transforms irreducibly according to $\Gamma_{n}$. Representations of the same dimension are distinguished by primes. Only two of the polynomials listed for $\Gamma_{2}$ are linearly independent.

\section{A. Discrete velocity sets}

The smallest discrete velocity sets invariant under the group of rotations of the cube are formed from the vectors describing the edges $\mathrm{E}$, vertices $\mathrm{V}$, faces $\mathrm{F}$, and the center $\mathrm{O}$ of a 
cube. Explicitly, these sets are

$$
\begin{aligned}
& 12 \text { edges } \quad E\{( \pm 1, \pm 1,0),( \pm 1,0, \pm 1),(0, \pm 1, \pm 1)\} \\
& 8 \text { vertices } \vee\{( \pm 1, \pm 1, \pm 1)\} \\
& 6 \text { faces } F\{( \pm 1,0,0),(0, \pm 1,0),(0,0, \pm 1)\} \\
& 1 \text { center } O\{(0,0,0)\}
\end{aligned}
$$

where, in the interest of simplicity, the lattice constant $\delta_{x}$ has been set to one. We consider these vectors as bases of vector spaces on which the group of rotations of the cube acts as a group of linear transformation and will also denote these representations by $\mathrm{E}, \mathrm{V}, \mathrm{F}$, and $\mathrm{O}$. Routine calculations $[14,15,24]$ give the decompositions into irreducible representations

$$
\begin{aligned}
& \mathrm{E}=\Gamma_{1} \oplus \Gamma_{2} \oplus 2 \Gamma_{3} \oplus \Gamma_{3}^{\prime}, \\
& \mathrm{V}=\Gamma_{1} \oplus \Gamma_{1}^{\prime} \oplus \Gamma_{3} \oplus \Gamma_{3}^{\prime}, \\
& \mathrm{F}=\Gamma_{1} \oplus \Gamma_{2} \oplus \Gamma_{3}^{\prime}, \\
& \mathrm{O}=\Gamma_{1}
\end{aligned}
$$

The explicit linear combinations of velocities that occur in these decompositions are listed in Appendix A.

The meaning of these decompositions is very simple. Consider, for example, the decomposition of $\mathrm{F}$ : if we have any linear combination of these velocities, say $\sum_{\boldsymbol{c}_{i} \in \mathrm{F}} a_{i} \boldsymbol{c}_{i}$, in terms of the basis vectors given in Eq. (A1), which we write as $\tilde{\boldsymbol{c}}^{1}, \tilde{\boldsymbol{c}}_{1}^{2}, \tilde{\boldsymbol{c}}_{2}^{2}, \tilde{\boldsymbol{c}}_{1}^{3}, \tilde{\boldsymbol{c}}_{2}^{3}, \tilde{\boldsymbol{c}}_{3}^{3}$, we have

$$
\sum_{\boldsymbol{c}_{i} \in \mathrm{F}} a_{i} \boldsymbol{c}_{i}=a^{1} \tilde{\boldsymbol{c}}^{1}+a_{1}^{2} \tilde{\boldsymbol{c}}_{1}^{2}+a_{2}^{2} \tilde{\boldsymbol{c}}_{2}^{2}+a_{1}^{3} \tilde{\boldsymbol{c}}_{1}^{3}+a_{2}^{3} \tilde{\boldsymbol{c}}_{2}^{3}+a_{3}^{3} \tilde{\boldsymbol{c}}_{3}^{3}
$$

Whereas arbitrary rotations of the sphere mix all of the coefficients $a_{i}, a^{1}$ is invariant under all rotations of the cube, the $a_{i}^{3}, i=1,2,3$ transform among themselves according to the irreducible representation $\Gamma_{3}^{\prime}$, and the $a_{i}^{2}$ transform according to $\Gamma_{2}$.

The absence of the representation $\Gamma_{3}$ in the decomposition of $F$ in Eq. (8c) suggests that the three polynomials $*\{x y\}$ that transform among themselves by $\Gamma_{3}$ according to Eq. (6), must vanish on the set F. Thus, this easily verified property has a group-theoretic significance. Similarly, the absence of the representation $\Gamma_{2}$ in the decomposition of $\mathrm{V}$ implies that $*\left\{x^{2}-y^{2}\right\}$ vanish on $\mathrm{V}$. Although these results are both obvious, later results will demonstrate that group theory can reveal much less obvious linear relations. 
Turning next to the velocity sets that are commonly used to construct LBE models, let us consider the D3Q13 [26], D3Q15, D3Q19, and D3Q27 models. The decompositions into irreducible representations of the representations of the group of rotations of the cube acting on the vector spaces generated by these velocity sets are perhaps best given in a table, which is easily obtained from Eqs. (8):

$$
\begin{array}{lll}
\text { model } & \text { velocity set } & \multicolumn{1}{c}{\text { decomposition }} \\
\hline \text { D3Q13 } & \mathrm{E} \cup \mathrm{O} & 2 \Gamma_{1} \oplus \Gamma_{2} \oplus 2 \Gamma_{3} \oplus \Gamma_{3}^{\prime} \\
\text { D3Q15 } & \mathrm{F} \cup \mathrm{V} \cup \mathrm{O} & 3 \Gamma_{1} \oplus \Gamma_{1}^{\prime} \oplus \Gamma_{2} \oplus \Gamma_{3} \oplus 2 \Gamma_{3}^{\prime} \\
\text { D3Q19 } & \mathrm{F} \cup \mathrm{E} \cup \mathrm{O} & 3 \Gamma_{1} \oplus 2 \Gamma_{2} \oplus 2 \Gamma_{3} \oplus 2 \Gamma_{3}^{\prime} \\
\text { D3Q21 } & \mathrm{E} \cup \mathrm{V} \cup \mathrm{O} & 3 \Gamma_{1} \oplus \Gamma_{1}^{\prime} \oplus \Gamma_{2} \oplus 3 \Gamma_{3} \oplus 2 \Gamma_{3}^{\prime} \\
\text { D3Q27 } \mathrm{E} \cup \mathrm{V} \cup \mathrm{F} \cup \mathrm{O} & 4 \Gamma_{1} \oplus \Gamma_{1}^{\prime} \oplus 2 \Gamma_{2} \oplus 3 \Gamma_{3} \oplus 3 \Gamma_{3}^{\prime}
\end{array}
$$

The theoretically possible model D3Q21 is given in the interest of completeness.

\section{B. Polynomials}

We next consider the polynomials $\left\{p_{j}(\boldsymbol{\xi})\right\}$ that generate the moments. The rotations of the cube form a group of linear transformations of the continuous vector variable $\boldsymbol{\xi}$, and by obvious extension, a group of linear transformations of homogeneous polynomials in the components of $\boldsymbol{\xi}$. We will again require the decomposition of these representations into irreducible representations.

Denote by $P^{n}$ the set of all homogeneous polynomials in $(x, y, z)$ of degree $n$. It is obvious that any constant $P^{0}$ is a rotational invariant and therefore transforms as $\Gamma_{1}$. According to Eq. (6), linear polynomials $P^{1}$ transform irreducibly as $\Gamma_{3}^{\prime}$. For quadratic polynomials, a new possibility arises: any quadratic polynomial is a linear combination of

$$
r^{2} \equiv x^{2}+y^{2}+z^{2}
$$

an invariant (also of the group of all rotations of space $\mathrm{SO}_{3}$ ), that transforms as $\Gamma_{1}$, and a remainder. This decomposition also occurs in the continuous case, where it is reflected in the occurrence of the (scalar) pressure as the trace of the stress tensor. This decomposition can be written as

$$
P^{2}=r^{2} P^{0} \oplus P^{2,0}
$$


where $P^{2,0}$ denotes the set $\left\{a_{i j} x_{i} x_{j} \mid a_{k k}=0\right\}$ of quadratics with a trace-free coefficient matrix. According to Eq. (8), the representation of the group of rotations of the cube on $P^{2,0}$ splits into the sum

$$
P^{2,0}=\Gamma_{2} \oplus \Gamma_{3}
$$

so that finally

$$
P^{2}=\Gamma_{1} \oplus \Gamma_{2} \oplus \Gamma_{3}
$$

For cubic polynomials, we note first that the product of $r^{2}$ and a linear polynomial evidently transforms by the irreducible representation $\Gamma_{3}^{\prime}$. Separating this contribution, we obtain an analog of Eq. (12)

$$
P^{3}=r^{2} P^{1} \oplus P^{3,0}
$$

The first term corresponds to the possibility of generating a vector from a symmetric thirdrank tensor $a_{i m n}$ by the contraction $a_{i}=\delta_{m n} a_{i m n}$. A familiar physical example is the generation of the heat flux vector from the stress flux tensor. The remaining 7 polynomials $P^{3,0}$ define an irreducible representation of $\mathrm{SO}_{3}$ on the vector space of cubic polynomials $\left\{a_{i m n} x_{i} x_{m} x_{n} \mid a_{i m n} \delta_{m n}=a_{i m n} \delta_{i n}=a_{i m n} \delta_{i m}=0\right\}$. Equations (12) and (15) are simple illustrations of the representation theory of $\mathrm{SO}_{3}$ as beautifully explained by Weyl [27].

The representation of the group of rotations of the cube on $P^{3,0}$ splits into the sum of irreducible representations

$$
P^{3,0}=\Gamma_{1}^{\prime} \oplus \Gamma_{3} \oplus \Gamma_{3}^{\prime}
$$

where the representations on the right side occur on the polynomials

$$
\begin{aligned}
& \Gamma_{1}^{\prime}: x y z \\
& \Gamma_{3}:\left\{* x\left(y^{2}-z^{2}\right)\right\} \\
& \Gamma_{3}^{\prime}:\left\{* x\left(2 x^{2}-3 y^{2}-3 z^{2}\right)\right\} .
\end{aligned}
$$

Since this decomposition is less obvious than the simple result for second rank tensors, we note that the decomposition in Eq. (16) is found using the character table, and that that vectors in Eq. (17) can be constructed systematically using projection operations [14, 15, 24]. The elementary calculations are not given here. It follows from Eqs. (15) and (16) that

$$
P^{3}=\Gamma_{1}^{\prime} \oplus \Gamma_{3} \oplus 2 \Gamma_{3}^{\prime}
$$

It may be surprising that a cubic polynomial $x\left(y^{2}-z^{2}\right)$ appears in a representation $\Gamma_{3}$ corresponding to a second rank tensor. This circumstance can perhaps be explained by a 
simple table as follows:

$$
\begin{array}{rrr|rrr|rrr}
x & y & z & y z & z x & x y & x\left(y^{2}-z^{2}\right) & y\left(z^{2}-x^{2}\right) & z\left(x^{2}-y^{2}\right) \\
-x & z & y & y z & -y x & -x z & x\left(y^{2}-z^{2}\right) & -z\left(x^{2}-y^{2}\right) & -y\left(z^{2}-x^{2}\right)
\end{array}
$$

The first three columns represent the symmetry operation $(x, y, z) \rightarrow(-x, z, y)$. The second three columns show the corresponding transformation of the three variables $*(y z)$ : comparing these two lines shows that the irreducible representations $\Gamma_{3}^{\prime}$ and $\Gamma_{3}$ 'represent' this symmetry operation by distinct transformations. The final three columns exhibit the transformation of the quantities $*\left\{x\left(y^{2}-z^{2}\right)\right\}$ : they obviously transform like the variables $*\{y z\}$, that is, by $\Gamma_{3}$ rather than by $\Gamma_{3}^{\prime}$. In the complete group of 48 symmetries of the cube, these two occurrences of $\Gamma_{3}$ would split into two representations with opposite parity under inversion. This point will be discussed further later in the paper.

For homogeneous quartic polynomials

$$
P^{4}=r^{4} P^{0} \oplus r^{2} P^{2,0} \oplus P^{4,0}
$$

For the group of rotations of the cube,

$$
P^{4,0}=\Gamma_{1} \oplus \Gamma_{2} \oplus \Gamma_{3} \oplus \Gamma_{3}^{\prime}
$$

where the representations on the right side occur on the polynomials

$$
\begin{aligned}
& \Gamma_{1}:\left\{I_{4}\right\} \\
& \Gamma_{2}:\left\{* 3\left(x^{4}-6 x^{2} y^{2}+y^{4}\right)-2 I_{4}\right\} \\
& \Gamma_{3}^{\prime}:\left\{* x y\left(x^{2}-y^{2}\right)\right\} \\
& \Gamma_{3}:\left\{* y z\left(6 x^{2}-y^{2}-z^{2}\right)\right\}
\end{aligned}
$$

where $I_{4}:=\left(x^{4}+y^{4}+z^{4}\right)-3\left(x^{2} y^{2}+y^{2} z^{2}+z^{2} x^{2}\right)$.

To summarize: we begin with the decompositions given by Eqs. (12), (15), and (20) of the homogeneous polynomials $P^{2}, P^{3}$, and $P^{4}$ of degrees 2,3 , and 4 into multiples of powers of invariant $r^{2}$, and the remaining polynomials $P^{2,0}, P^{3,0}$, and $P^{4,0}$ that are not multiples of powers of $r^{2}$. The representations of $\mathrm{SO}_{3}$ on the vector spaces $P^{2,0}, P^{3,0}$, and $P^{4,0}$ are well-known to be irreducible [27], but the representations of the group of rotations of the cube on these spaces are reducible, and their decompositions into irreducible representations are given as Eqs. (13), (16), and (21), in terms of the explicit polynomial sets defined in Eqs. (6), (17), and (22). The reducibility of these representations explains the possibility of incomplete moments; this connection will be developed in the next section. 


\section{THE MATRIX A - CONSTRUCTION OF MODELS}

In Sec. II A, a basis $\left\{\tilde{\boldsymbol{c}}_{i}\right\}$ of the discrete velocity space $C$ is constructed in which the basis elements belong to irreducible representations of the group of rotations of the cube; the corresponding basis of polynomials $\left\{\tilde{p}_{j}\right\}$ is constructed in Sec. II B. The velocities and polynomials are now combined by evaluating the matrix A using these bases; we recall that the result will be denoted by $\tilde{\mathrm{A}}$ and that the justification for introducing these bases is that all entries of $\tilde{\mathrm{A}}$ vanish unless $\left\{\tilde{\boldsymbol{c}}_{i}\right\}$ and $\left\{\tilde{\boldsymbol{p}}_{j}\right\}$ belong to the same irreducible representation.

The orthogonality properties of irreducible representations imply another simple but useful conclusion: assume that the set of velocities $C$ has been chosen and that the corresponding representation admits the decomposition into irreducible representations

$$
\mathrm{C}=n_{1} \Gamma_{1} \oplus n_{1}^{\prime} \Gamma_{1}^{\prime} \oplus n_{2} \Gamma_{2} \oplus n_{3} \Gamma_{3} \oplus n_{3}^{\prime} \Gamma_{3}^{\prime}
$$

In order that $A$ have full rank, it is necessary that representation on the polynomial set $\mathrm{P}$ admits exactly the same decomposition

$$
\mathrm{P}=n_{1} \Gamma_{1} \oplus n_{1}^{\prime} \Gamma_{1}^{\prime} \oplus n_{2} \Gamma_{2} \oplus n_{3} \Gamma_{3} \oplus n_{3}^{\prime} \Gamma_{3}^{\prime}
$$

Equations (23) and (24) refine the obvious condition that the velocity and polynomial sets must have the same dimension

$$
\operatorname{dim} \mathrm{C}=\operatorname{dim} \mathrm{P}=n_{1}+n_{1}^{\prime}+2 n_{2}+3 n_{3}+3 n_{3}^{\prime}
$$

The condition is only necessary, because nothing prevents the vanishing of a block $\left\langle\tilde{\boldsymbol{c}}_{i} \mid \tilde{\boldsymbol{p}}_{i}\right\rangle$; the rank of these blocks also must be checked. The process is best explained by examples.

\section{A. The D3Q6 model}

The simplest discrete model is Broadwell's original model with six velocities [28] based on the faces of a cube. Given the velocity set $F$ from Eq. (8), the problem is to choose polynomials to generate appropriate moments. This choice will be exhibited in tabular form 
as follows:

\begin{tabular}{l|lllll} 
& $\Gamma_{1}$ & $\Gamma_{1}^{\prime}$ & $\Gamma_{2}$ & $\Gamma_{3}$ & $\Gamma_{3}^{\prime}$ \\
\hline \hline D3Q6 & 1 & 0 & 1 & 0 & 1 \\
\hline$P^{0}$ & 1 & 0 & 0 & 0 & 0 \\
$P^{1}$ & 0 & 0 & 0 & 0 & 1 \\
$P^{2,0}$ & 0 & 0 & 1 & 1 & 0
\end{tabular}

The first row, labeled 'D3Q6,' shows the multiplicities of the irreducible representations for the velocity set $F$. The decompositions of the representations on polynomials of degrees 0 , 1 , and 2 are given on the next lines. But it is understood that $P^{0}$ can be any scalar $r^{2 n}$ and $P^{1}$ can be any vector $r^{2 n}\{* x\}$, therefore the next line corresponds to trace-free second rank tensors as in Eq. (12). This convention will also apply later to tensors of higher rank. Each row represents a complete moment, so that the goal is to choose entire rows as nearly as possible to construct the model.

Comparison of the first row with the others shows immediately that the model must contain contributions from each row; however, the complete trial set $P^{0} \oplus P_{1} \oplus P_{2}^{*}$, corresponding to a scalar, a vector, and a trace-free second-rank tensor, has dimension 9 . The explanation is that, as noted earlier, the quadratic polynomials that transform as $\Gamma_{3}, *\{x y\}$ vanish identically on the set F. We cannot include the complete polynomials $P^{2,0}$ to form the model, but are forced instead to select only the occurrence of $\Gamma_{2}$. The model based on D3Q6 therefore necessarily contains an incomplete second order moment [31].

The set of polynomials corresponding to the velocity set $\mathrm{F}$ must be

$$
\text { D3Q6 }\left\{\begin{array}{l}
\Gamma_{1} \text { from } P^{0}:\{1\} \\
\Gamma_{3}^{\prime} \text { from } P^{1}:\{* x\} \\
\Gamma_{2} \text { from } P^{2,0}:\left\{*\left(x^{2}-y^{2}\right)\right\}
\end{array}\right.
$$

It remains to verify that the polynomials actually are linearly independent over the discrete velocity set. In this case, the verification amounts to showing that no polynomial set vanishes identically on the set of points that transforms by the same irreducible representation. The trivial verification is omitted. 


\section{B. The D3Q15 model}

The table corresponding to Eq. (25) is

\begin{tabular}{l|lllll} 
& $\Gamma_{1}$ & $\Gamma_{1}^{\prime}$ & $\Gamma_{2}$ & $\Gamma_{3}$ & $\Gamma_{3}^{\prime}$ \\
\hline \hline D3Q15 & 3 & 1 & 1 & 1 & 2 \\
\hline$P^{0}$ & 1 & 0 & 0 & 0 & 0 \\
$P^{1}$ & 0 & 0 & 0 & 0 & 1 \\
$P^{2,0}$ & 0 & 0 & 1 & 1 & 0 \\
$P^{3,0}$ & 0 & 1 & 0 & 1 & 1
\end{tabular}

$P^{3,0}$ must be included in order to accommodate the occurrence of $\Gamma_{1}^{\prime}$ in D3Q15. However, the table immediately shows that the moment $P^{3,0}$, a pair-trace-free tensor of rank 3 , cannot be complete, because it will generate a redundant occurrence of $\Gamma_{3}$. As in the D3Q6 model, this issue can be identified by simply computing dimensions; the group theory analysis refines this observation by showing that a model based on the set D3Q15 must contain an incomplete third order moment. A polynomial set that generates the required irreducible representations is

$$
\text { D3Q15 }\left\{\begin{array}{l}
3 \Gamma_{1}:\{1\},\left\{r^{2}\right\},\left\{r^{4}\right\} \\
2 \Gamma_{3}^{\prime}:\{* x\},\left\{* x r^{2}\right\} \\
\Gamma_{2}:\left\{*\left(x^{2}-y^{2}\right)\right\} \\
\Gamma_{3}:\{* x y\} \\
\Gamma_{1}^{\prime}:\{x y z\}
\end{array}\right.
$$

The table of representations again suggests the degeneracies that must occur in this model. We have seen that the complete cubic moment generates a redundant occurrence of $\Gamma_{3}$, and indeed, the cubics that transform as $\Gamma_{3}$, given in Eq. (17) as $\left\{* x\left(y^{2}-z^{2}\right)\right\}$, all vanish identically on the velocity set D3Q15. The remaining cubics $* x\left(2 x^{2}-3 y^{2}-3 z^{2}\right)$ that transform as $\Gamma_{3}^{\prime}$, prove to be linearly dependent on the polynomials selected in Eq. (28), but we omit the simple verification.

We must again verify that these polynomials are linearly independent over the discrete set of velocities $\mathrm{F} \cup \mathrm{V} \cup \mathrm{O}$ that defines this model. For the irreducible representations that only occur once, it is sufficient to verify that the polynomials do not vanish identically. In this case, these representations are $\Gamma_{2}$ and $\Gamma_{3}$, corresponding to quadratic polynomials. We omit the easy verification. A nontrivial problem arises only for the irreducible representations that 
occur with multiplicity greater than one. Let us verify directly that the three polynomials that transform according to $\Gamma_{1}$ are linearly independent in this model. Using the results of Appendix A, the identity representation occurs on the center of the cube (the zero vector) and on $\frac{1}{6} \sum_{\boldsymbol{c}_{i} \in \mathrm{F}} \boldsymbol{c}_{i}$ and $\frac{1}{8} \sum_{\boldsymbol{c}_{i} \in \mathrm{V}} \boldsymbol{c}_{i}$. Evaluating the polynomials $1, r^{2}$, and $r^{4}$ on these three velocity vectors gives the matrix

$$
\left[\begin{array}{lll}
1 & 0 & 0 \\
1 & 1 & 1 \\
1 & 3 & 9
\end{array}\right] .
$$

Since this matrix is nonsingular, the required linear independence is demonstrated. A similar argument applies to the two occurrences of the representation $\Gamma_{3}^{\prime}$ and establishes that the polynomials in Eq. (28) are indeed linearly independent over the velocity set D3Q15.

The distribution function of this model is the finite sum

$$
f(\boldsymbol{x}, \boldsymbol{\xi}, t)=\sum_{\boldsymbol{c}_{i} \in \mathrm{F} \cup \mathrm{V} \cup \mathrm{O}} f_{i}(\boldsymbol{x}, t) \delta\left(\boldsymbol{\xi}-\boldsymbol{c}_{i}\right) .
$$

We have verified that the following nonvanishing linearly independent moments are possible in this model:

$$
\begin{aligned}
\rho & =\langle 1 \mid f\rangle, \\
e & =\left\langle r^{2} \mid f\right\rangle, \\
\varepsilon & =\left\langle r^{4} \mid f\right\rangle, \\
j & =\langle * x \mid f\rangle, \\
\boldsymbol{q} & =\left\langle * x r^{2} \mid f\right\rangle, \\
\mathrm{p} & =\left\langle *\left(x^{2}-y^{2}\right), * x y \mid f\right\rangle, \\
\mathrm{T} & =\langle x y z \mid f\rangle .
\end{aligned}
$$

The scalar moments are the mass density $\rho$, a quantity $e$ formally related to the internal energy, and a fourth order moment $\varepsilon$. The vector moments are the momentum $j$ and the vector $\boldsymbol{q}$ related to the energy flux. One second rank tensor moment exists, the momentum flux, or stress p. All of these moments are complete.

Again, we recall that in the Introduction we stated the restriction of this analysis to kinematics. By stating that the model contains the energy-flux related vector $\boldsymbol{q}$, we only assert that $\boldsymbol{q}$ is a contracted third-rank tensor that transforms properly as a vector; we do not assert that the D3Q15 model correctly models the heat flux. 


\section{The D3Q13 and D3Q19 models}

Without presenting the straightforward details, we note the moments in the D3Q13 model,

$$
\begin{aligned}
\rho & =\langle 1 \mid f\rangle, \\
e & =\left\langle r^{2} \mid f\right\rangle, \\
\boldsymbol{j} & =\langle * x \mid f\rangle, \\
\mathrm{p} & =\left\langle *\left(x^{2}-y^{2}\right), * x y \mid f\right\rangle, \\
\mathrm{T} & =\left\langle * x\left(y^{2}-z^{2}\right) \mid f\right\rangle,
\end{aligned}
$$

where the distribution function is

$$
f(\boldsymbol{x}, \boldsymbol{\xi}, t)=\sum_{\boldsymbol{c}_{i} \in \mathrm{E} \cup \mathrm{O}} f_{i}(\boldsymbol{x}, t) \delta\left(\boldsymbol{\xi}-\boldsymbol{c}_{i}\right) .
$$

The moment $\mathrm{T}$ is an incomplete third-rank tensor.

For the D3Q19 model, the moments are

$$
\begin{aligned}
& \rho=\langle 1 \mid f\rangle, \\
& e=\left\langle r^{2} \mid f\right\rangle, \\
& \varepsilon=\left\langle r^{4} \mid f\right\rangle, \\
& j=\langle * x \mid f\rangle, \\
& \boldsymbol{q}=\left\langle * x r^{2} \mid f\right\rangle, \\
& \mathrm{p}=\left\langle *\left(x^{2}-y^{2}\right), * x y \mid f\right\rangle, \\
& \mathrm{e}=\left\langle *\left(x^{2}-y^{2}\right) r^{2}, * x y r^{2} \mid f\right\rangle .
\end{aligned}
$$

where the distribution function is

$$
f(\boldsymbol{x}, \boldsymbol{\xi}, t)=\sum_{\boldsymbol{c}_{i} \in \mathrm{F} \cup \mathrm{E} \cup \mathrm{O}} f_{i}(\boldsymbol{x}, t) \delta\left(\boldsymbol{\xi}-\boldsymbol{c}_{i}\right) .
$$

In this model, the flux of $\boldsymbol{q}$ is the complete second-rank tensor e.

Whereas the D3Q13 and D3Q15 models both contain incomplete moments, all moments in the D3Q19 model are complete. This model is also observed to be numerically more stable than the D3Q15 model [29]. This suggests a possible connection between completeness of moments and numerical stability that may warrant further investigation, although at this point, such a connection is entirely conjectural. 


\section{The D3Q27 model}

We will not repeat the previous tabular procedure for choosing the polynomials: we merely recall from Eq. (10) that the points of the D3Q27 model decompose into $4 \Gamma_{1} \oplus \Gamma_{1}^{\prime} \oplus$ $2 \Gamma_{2} \oplus 3 \Gamma_{3} \oplus 3 \Gamma_{3}^{\prime}$. Consider the candidate polynomials

$$
\begin{aligned}
& 4 \Gamma_{1}:\{1\},\left\{r^{2}\right\},\left\{r^{4}\right\},\left\{r^{6}\right\}, \\
& \Gamma_{1}^{\prime}:\{x y z\}, \\
& 2 \Gamma_{2}:\left\{*\left(x^{2}-y^{2}\right)\right\},\left\{*\left(x^{2}-y^{2}\right) r^{2}\right\}, \\
& 3 \Gamma_{3}:\{* x y\},\left\{* x y r^{2}\right\},\left\{* x\left(y^{2}-z^{2}\right)\right\}, \\
& 3 \Gamma_{3}^{\prime}:\{* x\},\left\{* x r^{2}\right\},\left\{* x\left(2 x^{2}-3 y^{2}-3 z^{2}\right)\right\} .
\end{aligned}
$$

In this case, the moments are not linearly independent, although this is hardly obvious from the formulas themselves. The degeneracy proves to occur in the three occurrences of $\Gamma_{3}^{\prime}$. As in the analysis of the D3Q15 model, we must evaluate the nine polynomials $\{* x\},\left\{* x r^{2}\right\}$, $\left\{* x\left(2 x^{2}-3 y^{2}-3 z^{2}\right)\right\}$ on the nine velocity vectors

$$
\tilde{\boldsymbol{c}}_{\alpha}=\frac{1}{6} \sum_{\boldsymbol{c}_{i} \in \mathrm{F}} c_{i \alpha} \boldsymbol{c}_{i}, \quad \tilde{\boldsymbol{c}}_{\alpha}^{\prime}=\frac{1}{8} \sum_{\boldsymbol{c}_{i} \in \mathrm{V}} c_{i \alpha} \boldsymbol{c}_{i}, \quad \tilde{\boldsymbol{c}}_{\alpha}^{\prime \prime}=\frac{1}{8} \sum_{\boldsymbol{c}_{i} \in \mathrm{E}} c_{i \alpha} \boldsymbol{c}_{i}, \quad \alpha=1,2,3,
$$

on which $\Gamma_{3}^{\prime}$ occurs. We simply state the result of the calculation

$$
\left[\begin{array}{ccc}
\left\langle x_{\beta} \mid \tilde{\boldsymbol{c}}_{\alpha}\right\rangle & \left\langle r^{2} x_{\beta} \mid \tilde{\boldsymbol{c}}_{\alpha}\right\rangle & \left\langle x_{\beta}\left(2 x_{\beta}-3 x_{\beta+1}^{2}-3 x_{\beta+2}^{2}\right) \mid \tilde{\boldsymbol{c}}_{\alpha}\right\rangle \\
\left\langle x_{\beta} \mid \tilde{\boldsymbol{c}}_{\alpha}^{\prime}\right\rangle & \left\langle r^{2} x_{\beta} \mid \tilde{\boldsymbol{c}}_{\alpha}^{\prime}\right\rangle & \left\langle x_{\beta}\left(2 x_{\beta}-3 x_{\beta+1}^{2}-3 x_{\beta+2}^{2}\right) \mid \tilde{\boldsymbol{c}}_{\alpha}^{\prime}\right\rangle \\
\left\langle x_{\beta} \mid \tilde{\boldsymbol{c}}_{\alpha}^{\prime \prime}\right\rangle & \left\langle r^{2} x_{\beta} \mid \tilde{\boldsymbol{c}}_{\alpha}^{\prime \prime}\right\rangle & \left\langle x_{\beta}\left(2 x_{\beta}-3 c_{i \beta+1}^{2}-3 c_{i \beta+2}^{2}\right) \mid \tilde{\boldsymbol{c}}_{\alpha}^{\prime \prime}\right\rangle
\end{array}\right]=\left[\begin{array}{rrr}
2 \mathrm{I}_{3 \times 3} & 2 \mathrm{I}_{3 \times 3} & 4 \mathrm{I}_{3 \times 3} \\
8 \mathrm{I}_{3 \times 3} & 24 \mathrm{I}_{3 \times 3} & -32 \mathrm{I}_{3 \times 3} \\
8 \mathrm{I}_{3 \times 3} & 16 \mathrm{I}_{3 \times 3} & -8 \mathrm{I}_{3 \times 3}
\end{array}\right] .
$$

Since

$$
\operatorname{det}\left[\begin{array}{rrr}
2 & 2 & 4 \\
8 & 24 & -32 \\
8 & 16 & -8
\end{array}\right]=0
$$

we see that the three polynomials which transform as $\Gamma_{3}^{\prime}$ are not linearly independent on the set of velocities chosen.

Lallemand [8] observed that the linear independence could be restored simply by replacing the velocities corresponding to the faces by the vectors $\mathrm{F}^{*}=$ $\{( \pm 2,0,0),(0, \pm 2,0),(0,0, \pm 2)\}$. A routine calculation shows that the polynomials which transform as $\Gamma_{3}^{\prime}$ are indeed linearly independent over this set and that in fact all the moments defined by these polynomials are indeed independent. 
This model therefore contains the following linearly independent moments:

$$
\begin{aligned}
\rho & =\langle 1 \mid f\rangle, \\
e & =\left\langle r^{2} \mid f\right\rangle, \\
\varepsilon_{1} & =\left\langle r^{4} \mid f\right\rangle, \\
\varepsilon_{2} & =\left\langle r^{6} \mid f\right\rangle, \\
\boldsymbol{j} & =\langle * x \mid f\rangle, \\
\boldsymbol{q} & =\left\langle * x r^{2} \mid f\right\rangle, \\
\mathrm{p} & =\left\langle *\left(x^{2}-y^{2}\right), * x y \mid f\right\rangle, \\
\mathrm{e} & =\left\langle *\left(x^{2}-y^{2}\right) r^{2}, * x y r^{2} \mid f\right\rangle, \\
\mathrm{T} & =\left\langle x y z, * x\left(y^{2}-z^{2}\right), * x\left(2 x^{2}-3 y^{2}-3 z^{2}\right) \mid f\right\rangle .
\end{aligned}
$$

where the distribution function is

$$
f(\boldsymbol{x}, \boldsymbol{\xi}, t)=\sum_{\boldsymbol{c}_{i} \in 2 \mathrm{~F} \cup \mathrm{E} \cup \mathrm{V} \cup \mathrm{O}} f_{i}(\boldsymbol{x}, t) \delta\left(\boldsymbol{\xi}-\boldsymbol{c}_{i}\right)
$$

In this model, a fourth scalar $\varepsilon_{2}$ exists, and the third rank tensor $\mathrm{T}$ is complete. Eqs. (20) and (21) show that the set of all quartics decomposes into irreducible representations as $P^{4}=4 \Gamma_{1} \oplus \Gamma_{1}^{\prime} \oplus 3 \Gamma_{2} \oplus 4 \Gamma_{3} \oplus 4 \Gamma_{3}^{\prime}$ and contains excess occurrences of $\Gamma_{3}$ and $\Gamma_{3}^{\prime}$. We observe that the three polynomials $* x y\left(x^{2}-y^{2}\right)$ that transform as $\Gamma_{3}^{\prime}$ (compare Eq. (22)) vanish on all velocities D3Q27. It is straightforward to obtain the linear dependencies between the quartic velocities $\left\{* y z\left(6 x^{2}-y^{2}-z^{2}\right)\right\}$ and the velocities of the D3Q27 model.

\section{A POSSIBLE HIGHER ORDER MODEL - D3Q51}

We next consider a theoretically possible higher order model. We noted that the quartics $* x y\left(x^{2}-y^{2}\right)$ vanish on the discrete velocity sets based on the edges, vertices, faces, and center; therefore, in any model based on these sets or their multiples, fourth rank tensors cannot be complete. To obtain complete fourth rank tensors, we need a different kind of velocity set.

A general vector has 24 distinct images under the 24 rotations of the cube; the edges, faces, and vertices have fewer because they are invariant under special rotations. In order to avoid the linear dependencies of the special quartic polynomials, let 
us add the 24 velocities obtained as the transforms of $(2,1,0)$. This set, containing the velocities with the smallest energy beyond the D3Q27 model, is denoted as G = $\{( \pm 2, \pm 1,0),( \pm 1, \pm 2,0),( \pm 2,0, \pm 1),( \pm 1,0, \pm 2),(0, \pm 2, \pm 1),(0, \pm 1, \pm 2)\}$.

The representation of the group of rotations of the cube on this set decomposes into the irreducible representations

$$
\mathrm{G}=\Gamma_{1} \oplus \Gamma_{1}^{\prime} \oplus 2 \Gamma_{2} \oplus 3 \Gamma_{3} \oplus 3 \Gamma_{3}^{\prime}
$$

The representation on any set of 24 points obtained as the distinct images of one lattice point will always admit the same decomposition into irreducible representations as Eq. (41): this is the 'regular representation' of group theory.

Let us consider the discrete velocity set of the D3Q27 model augmented by this set, giving the velocity set $\mathrm{D} 3 \mathrm{Q} 51=\mathrm{O} \cup \mathrm{F} \cup \mathrm{V} \cup \mathrm{E} \cup \mathrm{G}$. The representation on this velocity set decomposes into irreducible representations as

$$
\mathrm{O} \oplus \mathrm{F} \oplus \mathrm{V} \oplus \mathrm{E} \oplus \mathrm{G}=5 \Gamma_{1} \oplus 2 \Gamma_{1}^{\prime} \oplus 4 \Gamma_{2} \oplus 6 \Gamma_{3} \oplus 6 \Gamma_{3}^{\prime}
$$

We again exhibit the choice of polynomials in tabular form:

\begin{tabular}{l|lllll} 
& $\Gamma_{1}$ & $\Gamma_{1}^{\prime}$ & $\Gamma_{2}$ & $\Gamma_{3}$ & $\Gamma_{3}^{\prime}$ \\
\hline \hline D3Q51 & 5 & 2 & 4 & 6 & 6 \\
\hline$P^{0}$ & 1 & 0 & 0 & 0 & 0 \\
$P^{1}$ & 0 & 0 & 0 & 0 & 1 \\
$P^{2,0}$ & 0 & 0 & 1 & 1 & 0 \\
$P^{3,0}$ & 0 & 1 & 0 & 1 & 1 \\
$P^{4,0}$ & 1 & 0 & 1 & 1 & 1 \\
$P^{5,0}$ & 0 & 0 & 1 & 1 & 2 \\
$P^{6,0}$ & 1 & 1 & 1 & 2 & 1
\end{tabular}

A trial set of polynomials can be constructed using polynomials up to degree $5,5 P^{0} \oplus$ $2 P^{1} \oplus 2 P^{2,0} \oplus 2 P^{3,0} \oplus P^{4,0} \oplus P^{5,0}$ where multiple occurrences of $P^{0}, P^{1}, P^{2,0}$, and $P^{3,0}$ are obtained from sets like $r^{2} P^{0}$ and $r^{2} P^{1}$. The table shows that the decomposition into irreducible representations for this polynomial set is $5 \Gamma_{1} \oplus 2 \Gamma_{1}^{\prime} \oplus 4 \Gamma_{2} \oplus 6 \Gamma_{3} \oplus 6 \Gamma_{3}^{\prime}$, which coincides with the decomposition of the velocity set. However, these polynomials are not linearly independent over the velocity set D3Q51. The problem is with the representation 
$\Gamma_{1}^{\prime}$, which we see can only occur in $P^{3,0}$; to obtain two occurrences of $\Gamma_{1}^{\prime}$, we will also require the polynomials $r^{2} P^{3,0}$ so that $\Gamma_{1}^{\prime}$ will occur on the polynomials $x y z$ and $r^{2} x y z$. But both vanish on the sets $\mathrm{G}, \mathrm{F}$, and $\mathrm{E}$, and they coincide on $\mathrm{V}$. It follows that $x y z$ and $r^{2} x y z$ are not linearly independent on D3Q51, and the dimension of the polynomial set is in fact no more than 50 .

We can try to solve the problem by using polynomials of degree 6 , for which a second representation $\Gamma_{1}^{\prime}$ appears. Explicitly, it is on the polynomial

$$
x^{2} y^{2}\left(x^{2}-y^{2}\right)+y^{2} z^{2}\left(y^{2}-z^{2}\right)+z^{2} x^{2}\left(z^{2}-x^{2}\right)
$$

which obviously does not vanish on the set $\mathrm{G}$. This polynomial, and $x y z$ give two independent polynomials that transform by $\Gamma_{1}^{\prime}$. However, the selection of the remaining polynomials is not straightforward: the trial set

$$
5 P^{0} \oplus P^{1} \oplus P^{2,0} \oplus P^{3,0} \oplus P^{4,0} \oplus P^{5,0} \oplus P^{6,0}
$$

has the same decomposition into irreducible representations as the velocity set D3Q51, but the appearance of only a single $P^{1}$ means that a contracted tensor of the form $r^{2} P_{1}^{*}$, which is needed to represent the heat flux, does not appear. On the other hand, returning to the previous trial set but replacing $r^{2} x y z$ with the sixth degree polynomial noted above will create an incomplete third rank tensor. While this example underscores some of the difficulty of constructing higher dimensional discrete models, it also shows how group theory can help identify problems relatively quickly.

\section{TWO-DIMENSIONAL MODELS}

To treat two-dimensional models by the group theoretic approach, the symmetry group of the cube is replaced by a symmetry group of the square. It will be taken to be the group of eight transformations: the four $90^{\circ}$ rotations, and the four reflections through the diagonals and the bisectors of opposite sides. This group is larger than the group of four rotations of the square, and is chosen to provide as many representations as possible. The choice of the symmetry group will be discussed further in the next section. 
The irreducible representations are

\begin{tabular}{l|l}
\hline repr. & polynomials \\
\hline$\Gamma_{1}$ & 1 \\
$\Gamma_{2}$ & $x, y$ \\
$\Gamma_{1}^{\prime}$ & $\left(x^{2}-y^{2}\right)$ \\
$\Gamma_{1}^{\prime \prime}$ & $x y$ \\
$\Gamma_{1}^{\prime \prime \prime}$ & $x y\left(x^{2}-y^{2}\right)$ \\
\hline
\end{tabular}

The simplest sets of discrete velocities invariant under this symmetry group are formed from the faces and vertices of the square, to which we add as before the center,

$$
\begin{aligned}
& \mathrm{F}=\{(1,0),(0,1),(-1,0),(0,-1)\}, \\
& \mathrm{V}=\{(1,1),(-1,1),(-1,-1),(1,-1)\}, \\
& \mathrm{O}=\{(0,0)\}
\end{aligned}
$$

The corresponding representations decompose into irreducible representations as

$$
\begin{aligned}
\mathrm{F} & =\Gamma_{1} \oplus \Gamma_{2} \oplus \Gamma_{1}^{\prime}, \\
\mathrm{V} & =\Gamma_{1} \oplus \Gamma_{2} \oplus \Gamma_{1}^{\prime \prime}, \\
\mathrm{O} & =\Gamma_{1} .
\end{aligned}
$$

As in the symmetry group of the cube, the representation of the continuous rotation group on trace-free second rank tensors, given as the representation on quadratics $\left\{\left(x^{2}-y^{2}\right), 2 x y\right\}$ splits into $\Gamma_{1}^{\prime} \oplus \Gamma_{1}^{\prime \prime}$. To describe a second rank tensor completely, both representations are needed.

The simplest model in which a second rank tensor is complete D2Q9, defined by the set $\mathrm{F} \cup \mathrm{V} \cup \mathrm{O}$. Since the representation on these velocities decomposes into

$$
\mathrm{F} \oplus \mathrm{V} \oplus \mathrm{O}=3 \Gamma_{1} \oplus 2 \Gamma_{2} \oplus \Gamma_{1}^{\prime} \oplus \Gamma_{1}^{\prime \prime}
$$

we choose candidate polynomials belonging to the same representations,

$$
\begin{aligned}
& 3 \Gamma_{1}:\{1\},\left\{r^{2}\right\},\left\{r^{4}\right\}, \\
& 2 \Gamma_{2}:\{x, y\},\left\{x r^{2}, y r^{2}\right\}, \\
& \Gamma_{1}^{\prime}: x^{2}-y^{2} \\
& \Gamma_{1}^{\prime \prime}: x y .
\end{aligned}
$$


These moments are easily shown to be linearly independent over the defining velocity set; accordingly, this 9-velocity model describes the following moments:

$$
\begin{aligned}
\rho & =\langle 1 \mid f\rangle, \\
e & =\left\langle r^{2} \mid f\right\rangle, \\
\varepsilon_{1} & =\left\langle r^{4} \mid f\right\rangle, \\
\boldsymbol{j} & =\langle x, y \mid f\rangle, \\
\boldsymbol{q} & =\left\langle r^{2} x, r^{2} y \mid f\right\rangle, \\
\mathrm{p} & =\left\langle x^{2} y^{2}, x y \mid f\right\rangle .
\end{aligned}
$$

To obtain a complete third rank tensor, we can add the velocities $\mathrm{F}^{*}=$ $\{(2,0),(0,2),(-2,0),(0,-2)\}$ resulting in a model D2Q13. The representation on the velocity set $\mathrm{F} \cup 2 \mathrm{~F} \cup \mathrm{V} \cup \mathrm{O}$ decomposes into

$$
\mathrm{F} \oplus 2 \mathrm{~F} \oplus \mathrm{V} \oplus \mathrm{O}=4 \Gamma_{1} \oplus 3 \Gamma_{2} \oplus 2 \Gamma_{1}^{\prime} \oplus \Gamma_{1}^{\prime \prime}
$$

The polynomial set

$$
\begin{aligned}
& 4 \Gamma_{1}:\{1\},\left\{r^{2}\right\},\left\{r^{4}\right\},\left\{r^{6}\right\} \\
& 3 \Gamma_{2}:\{x, y\},\left\{x r^{2}, y r^{2}\right\},\left\{x\left(x^{2}-3 y^{2}\right), y\left(3 x^{2}-y^{2}\right)\right\} \\
& 2 \Gamma_{1}^{\prime}:\left(x^{2}-y^{2}\right),\left(x^{2}-y^{2}\right) r^{2} \\
& \Gamma_{1}^{\prime \prime}: x y
\end{aligned}
$$

is easily shown to be linearly independent over the chosen finite velocity space.

Let us briefly consider the formulation of higher order models. Eight more velocities can be added to $F, V$, and $\mathrm{O}$, either as the set $\left.\mathrm{F}^{*} \cup \mathrm{V}^{*}=\{ \pm 2,0),(0, \pm 2),( \pm 2, \pm 2)\right\}$, or, following the D3Q51 model, as the set $G=\{( \pm 2, \pm 1),( \pm 1, \pm 2)\}$. The first set leads to a 17-velocity model in 2D, which like D2Q9, cannot describe a complete fourth rank tensor: the polynomial $x y\left(x^{2}-y^{2}\right)$ still vanishes on all of the discrete velocities. On the other hand, the representation on $\mathrm{G}$ decomposes as $\mathrm{G}=\Gamma_{1} \oplus \Gamma_{1}^{\prime} \oplus \Gamma_{1}^{\prime \prime} \oplus \Gamma_{1}^{\prime \prime \prime} \oplus 2 \Gamma_{2}$. The resulting D2Q17 model contains a complete fourth rank tensor, but we do not present the details, which should now be obvious. 


\section{THE CHOICE OF THE SYMMETRY GROUP}

In this paper, the only role of the symmetry group of the velocity set is to help simplify the calculation of the matrix A; no particular physical importance is attached to it. Thus, the group of rotations of a cube was chosen simply for ease of exposition. It might seem physically more natural to choose the group of 48 symmetries that includes the inversions $(x, y, z) \rightarrow(-x,-y,-z)$. The representation theory of this group yields two irreducible representations with opposite parity under inversions for every irreducible representation of the group of 24 rotations: one defined by even order polynomials, and the other defined by odd order polynomials. But the orthogonality properties of irreducible representations are all that we require; since polynomials of opposite parity are automatically orthogonal, no additional simplifications would result from explicitly introducing the group of 48 symmetries.

The situation is quite different for the two-dimensional models. If we had chosen the group of four rotations of a square as the basic group, there would only be four irreducible representations. By choosing a larger group with more irreducible representations, we can increase the number of vanishing elements in the matrix $A$ thereby lightening the algebra.

\section{HIGHER ORDER MOMENTS IN LBE MODELS}

In the usual hydrodynamics described by the Navier-Stokes equations, the highest rank tensor involved is two; the third rank stress flux is reduced by index contraction to the heat flux vector $\boldsymbol{q}$. But a generalized hydrodynamics model, such as Grad's 13-moment model [30], might attempt to model the complete stress flux. In this case, the model should contain complete moments up to order at least three. But since the advection operator couples moments to their fluxes, which are of order one greater, a model of the stress flux should contain complete tensors of ranks up to four. More generally, for the linearized Boltzmann equation or the Maxwell molecules, moments must be complete at least to order $N+1$ for a dynamically realistic model of moments of order $N$. Modeling of higher order moments could be of interest for LBE simulations of certain special cases of finite Knudsen number flows.

It must be emphasized that we are only interested in the linearized Boltzmann equation, 
on which the multiple relaxation time formalism, and indeed most other lattice Boltzmann models, are based; therefore, higher order moments generated by the nonlinear collision operator are not considered.

\section{CONCLUSIONS}

The representation theory of finite groups has been applied to the systematic construction of Lattice Boltzmann models. Given the choice of a finite velocity set $\left\{\boldsymbol{c}_{i}\right\}$, the method helps identify the moments for which a kinematically satisfactory description is possible. While these moments can be identified by elementary means, group theory simplifies the calculations by introducing natural vector space bases in which the calculations are particularly simple.

By increasing the dimension of the space of moments, adding more velocities obviously makes complete moments of higher order possible. Thus, in three dimensions, the Broadwell model based on six velocities contains an incomplete second rank tensor; in the D3Q15 and D3Q19 models, the second rank tensors are complete but a third rank tensor is incomplete; in the D3Q27 model, the lowest rank of an incomplete tensor is four. A similar progression occurred for the two-dimensional models. We used group theory to determine exactly which moments are complete; this may not be obvious from the velocity set itself. Some of the difficulties in choosing discrete velocities to achieve complete moments of a given order were discussed in Sec. IV.

We can compare the present approach with a straightforward orthogonalization procedure applied directly to the velocities. It is evident that the results of the group-theory selection of polynomials could be reproduced if one happened to make the appropriate choices in the orthogonalization. Group theory offers an advantage when the model is extended by adding new velocities, for whereas orthogonalization must be repeated from the beginning over the new velocity set, group theory makes it easy to generate polynomials that correspond to the irreducible representations introduced by the new velocities.

Finally, we should remark on a limitation of group theory, at least on the level discussed in this paper: analysis of the model in the method of Lallemand also requires that we compute the dispersion relation of waves in the finite wavenumber hydrodynamics $[5,8,9]$. In effect, this paper accomplishes this analysis only in the limit of zero wavenumber. The 
analysis for finite wavenumbers remains difficult, although perhaps a more sophisticated application of group theory might be useful in that problem as well. The finite wavenumber analysis can couple the lower and higher order moments; in this case, anisotropy of wave dispersion is possible if the higher order moments are incomplete. Thus, incomplete higher order moments can have consequences even for models that are restricted to Navier-Stokes hydrodynamics.

\section{APPENDIX A: SYMMETRY GROUP OF THE CUBE}

This appendix lists the decompositions into irreducible representations of the representations of the symmetry group of the cube on the faces, edges, and vertices.

Denote the vectors corresponding to the faces of the cube as $\boldsymbol{c}_{1}=(1,0,0), \boldsymbol{c}_{2}=$ $(-1,0,0), \boldsymbol{c}_{3}=(0,1,0), \boldsymbol{c}_{4}=(0,-1,0), \boldsymbol{c}_{5}=(0,0,1)$, and $\boldsymbol{c}_{6}=(0,0,-1)$. The decomposition into irreducible representations is given by the linear combinations

\begin{tabular}{r|rrrrrr}
$\mathrm{F}$ & $\boldsymbol{c}_{1}$ & $\boldsymbol{c}_{2}$ & $\boldsymbol{c}_{3}$ & $\boldsymbol{c}_{4}$ & $\boldsymbol{c}_{5}$ & $\boldsymbol{c}_{6}$ \\
\hline \hline$\Gamma_{1}$ & 1 & 1 & 1 & 1 & 1 & 1 \\
\hline & 1 & 1 & -1 & -1 & 0 & 0 \\
$\Gamma_{2}$ & 0 & 0 & 1 & 1 & -1 & -1 \\
& -1 & -1 & 0 & 0 & 1 & 1 \\
\hline \multirow{3}{*}{$\Gamma_{3}^{\prime}$} & 1 & -1 & 0 & 0 & 0 & 0 \\
& 0 & 0 & 1 & -1 & 0 & 0 \\
& 0 & 0 & 0 & 0 & 1 & -1
\end{tabular}

The entry corresponding to $\Gamma_{3}^{\prime}$ means that the three linear combinations $\left(\boldsymbol{c}_{1}-\boldsymbol{c}_{2}\right),\left(\boldsymbol{c}_{3}-\boldsymbol{c}_{4}\right)$, $\left(\boldsymbol{c}_{5}-\boldsymbol{c}_{6}\right)$ transform like a vector under the symmetry group of the cube. Note that the three linear combinations listed as transforming as $\Gamma_{2}$ are linearly dependent.

The linear combinations are of the form $\sum_{1 \leq i \leq 6} p_{j}\left(\boldsymbol{c}_{i}\right) \boldsymbol{c}_{i}$, where the $p_{j}$ are polynomials transforming irreducibly as $\Gamma_{1}, \Gamma_{2}$, and $\Gamma_{3}$. In Eq. (A1), these polynomials are, respectively, $\{1\},\left\{*\left(x^{2}-y^{2}\right)\right\}$, and $\{* x\}$.

Next, write the vectors corresponding to the eight vertices as $\boldsymbol{c}_{1}=(1,1,1), \boldsymbol{c}_{2}=$ $(1,1,-1), \boldsymbol{c}_{3}=(1,-1,1), \boldsymbol{c}_{4}=(-1,1,1), \boldsymbol{c}_{5}=(1,-1,-1), \boldsymbol{c}_{6}=(-1,1,-1)$, $\boldsymbol{c}_{7}=(-1,-1,1)$, and $\boldsymbol{c}_{8}=(-1,-1,-1)$. The decomposition into irreducible represen- 
tations is given by the linear combinations

\begin{tabular}{r|rrrrrrrr}
$\vee$ & $\boldsymbol{c}_{1}$ & $\boldsymbol{c}_{2}$ & $\boldsymbol{c}_{3}$ & $\boldsymbol{c}_{4}$ & $\boldsymbol{c}_{5}$ & $\boldsymbol{c}_{6}$ & $\boldsymbol{c}_{7}$ & $\boldsymbol{c}_{8}$ \\
\hline \hline$\Gamma_{1}$ & 1 & 1 & 1 & 1 & 1 & 1 & 1 & 1 \\
\hline$\Gamma_{1}^{\prime}$ & 1 & 1 & -1 & -1 & 1 & 1 & 1 & 1 \\
\hline & 1 & 1 & -1 & -1 & -1 & -1 & 1 & 1 \\
$\Gamma_{3}$ & 1 & -1 & -1 & 1 & 1 & -1 & -1 & 1 \\
& 1 & -1 & 1 & -1 & -1 & 1 & -1 & 1 \\
\hline & 1 & 1 & 1 & -1 & 1 & -1 & -1 & -1 \\
$\Gamma_{3}^{\prime}$ & 1 & 1 & -1 & 1 & -1 & 1 & -1 & -1 \\
& 1 & -1 & 1 & 1 & -1 & -1 & 1 & -1
\end{tabular}

Again, this table is written more succinctly as the linear combinations $\sum_{1 \leq i \leq 8} p_{j}\left(\boldsymbol{c}_{i}\right) \boldsymbol{c}_{i}$ where the $p_{j}$ are polynomials $\{1\},\{x y z\},\{* x y\}$, and $\{* x\}$.

Finally, denote the vectors corresponding to the edges as: $\boldsymbol{c}_{1}=(1,1,0), \boldsymbol{c}_{2}=(-1,1,0)$, $\boldsymbol{c}_{3}=(-1,-1,0), \boldsymbol{c}_{4}=(1,-1,0), \boldsymbol{c}_{5}=(0,1,1), \boldsymbol{c}_{6}=(0,-1,1), \boldsymbol{c}_{7}=(0,-1,-1)$, $\boldsymbol{c}_{8}=(0,1,-1), \boldsymbol{c}_{9}=(1,0,1), \boldsymbol{c}_{10}=(-1,0,1), \boldsymbol{c}_{11}=(-1,0,-1)$, and $\boldsymbol{c}_{12}=(1,0,-1)$.

The decomposition is

\begin{tabular}{l|rrrrrrrrrrrr}
$\mathrm{E}$ & $\boldsymbol{c}_{1}$ & $\boldsymbol{c}_{2}$ & $\boldsymbol{c}_{3}$ & $\boldsymbol{c}_{4}$ & $\boldsymbol{c}_{5}$ & $\boldsymbol{c}_{6}$ & $\boldsymbol{c}_{7}$ & $\boldsymbol{c}_{8}$ & $\boldsymbol{c}_{9}$ & $\boldsymbol{c}_{10}$ & $\boldsymbol{c}_{11}$ & $\boldsymbol{c}_{12}$ \\
\hline \hline$\Gamma_{1}$ & 1 & 1 & 1 & 1 & 1 & 1 & 1 & 1 & 1 & 1 & 1 & 1 \\
\hline$\Gamma_{2}$ & 2 & 2 & 2 & 2 & -1 & -1 & -1 & -1 & -1 & -1 & -1 & -1 \\
& -1 & -1 & -1 & -1 & 2 & 2 & 2 & 2 & -1 & -1 & -1 & -1 \\
& -1 & -1 & -1 & -1 & -1 & -1 & -1 & -1 & 2 & 2 & 2 & 2 \\
\hline \multirow{5}{*}{$\Gamma_{3}^{\prime}$} & 0 & 0 & 0 & 0 & 1 & 1 & -1 & -1 & 1 & 1 & -1 & -1 \\
& 1 & 1 & -1 & -1 & 1 & -1 & -1 & 1 & 0 & 0 & 0 & 0 \\
\hline & 1 & -1 & -1 & 1 & 0 & 0 & 0 & 0 & 1 & -1 & -1 & 1 \\
\hline & 0 & 0 & 0 & 0 & 1 & -1 & 1 & -1 & 0 & 0 & 0 & 0 \\
& 0 & 0 & 0 & 0 & 0 & 0 & 0 & 0 & 1 & -1 & 1 & -1 \\
& 1 & -1 & 1 & -1 & 0 & 0 & 0 & 0 & 0 & 0 & 0 & 0 \\
\hline & 1 & 1 & 1 & 1 & 0 & 0 & 0 & 0 & -1 & -1 & -1 & -1 \\
$\Gamma_{3}$ & -1 & -1 & -1 & -1 & 1 & 1 & 1 & 1 & 0 & 0 & 0 & 0 \\
& 0 & 0 & 0 & 0 & -1 & -1 & -1 & -1 & 1 & 1 & 1 & 1
\end{tabular}


These linear combinations are $\sum_{1 \leq i \leq 12} p_{j}\left(\boldsymbol{c}_{i}\right) \boldsymbol{c}_{i}$ where the $p_{j}$ are respectively $\{1\}\left(\Gamma_{1}\right)$, $\left\{*\left(x^{2}-y^{2}\right)\right\}\left(\Gamma_{2}\right),\{* x\}\left(\Gamma_{3}^{\prime}\right),\{* x y\}\left(\Gamma_{3}\right)$, and $\left\{* x\left(y^{2}-z^{2}\right)\right\}\left(\Gamma_{3}\right)$.

[1] X. He and L.-S. Luo, Phys. Rev. E 55, R6333 (1997).

[2] X. He and L.-S. Luo, Phys. Rev. E 56, 6811 (1997).

[3] L.-S. Luo, Phys. Rev. Lett. 81, 1618 (1998).

[4] L.-S. Luo, Phys. Rev. E 62, 4982 (2000).

[5] P. Lallemand and L.-S. Luo, Phys. Rev. E 61, 6546 (2000).

[6] L.-S. Luo and S. S. Girimaji, Phys. Rev. E 66, 035301(R) (2002).

[7] L.-S. Luo and S. S. Girimaji, Phys. Rev. E 67, 036302 (2003).

[8] P. Lallemand, D. d'Humières, L.-S. Luo, and R. Rubinstein, Phys. Rev. E 67, 021203 (2003).

[9] P. Lallemand and L.-S. Luo, Phys. Rev. E 68, 036706 (2003).

[10] M. Junk, A. Klar, and L.-S. Luo, J. Computat. Phys. 210, 676 (2005).

[11] S. Harris, An Introduction to the Theory of the Boltzmann Equation (Dover, Mineola, NY, 2004).

[12] J. O. Hirschfelder, C. F. Curtiss, and R. B. Bird, Molecular Theory of Gases and Liquids (Wiley, New York, 1954).

[13] D. d'Humières, I. Ginzburg, M. Krafczyk, P. Lallemand, and L.-S. Luo, Philos. Tran. R. Soc. Lond. A 360, 437 (2002).

[14] M. Hamermesh, Group Theory and Its Application to Physical Problems (Addison-Wesley, Reading, MA, 1962).

[15] J. F. Cornwell, Group Theory in Physics, vol. I (Academic Press, New York, 1984).

[16] S. Wolfram, J. Stat. Phys. 45, 471 (1986).

[17] P. Grosfils, Ph.D. thesis, Université de Bruxelles (1994).

[18] B. M. Boghosian and W. Taylor, Physica D 120, 30 (1998).

[19] B. M. Boghosian and W. Taylor, Phys. Rev. E 57, 54 (1998).

[20] M. Vergassola, R. Benzi, and S. Succi, Europhys. Lett. 13, 411 (1990).

[21] U. Frisch, Physica D 47, 231 (1991).

[22] P. J. Dellar, Phys. Rev. E 65, 036309 (2002).

[23] P. J. Dellar, J. Computat. Phys. 190, 351 (2003). 
[24] W. Fulton and J. Harris, Representation Theory: A First Course (Springer, New York, 1991).

[25] D. d'Humières, in Rarefied Gas Dynamics: Theory and Simulations, edited by B. D. Shizgal and D. P. Weave (AIAA, Washington, DC, 1992), vol. 159 of Prog. Astronaut. Aeronaut., pp. $450-458$.

[26] D. d'Humières, M. Bouzidi, and P. Lallemand, Phys. Rev. E 63, 066702 (2001).

[27] H. Weyl, The Classical Groups: Their Invariants and Representations (Princeton University Press, Princeton, NJ, 1953), 2nd ed.

[28] J. E. Broadwell, Phys. Fluids 7, 1243 (1964).

[29] R. Mei, W. Shyy, D. Yu, and L.-S. Luo, J. Computat. Phys. 161, 680 (2000).

[30] H. Grad, Comm. Pure Appl. Math. 2, 331 (1949).

[31] It should be noted that this fact was irrelevant to Broadwell's work. 